\title{
The effects of quantitative fecundity in the haploid stage on reproductive success and diploid fitness in the aquatic peat moss Sphagnum macrophyllum
}

\author{
MG Johnson ${ }^{1}$ and AJ Shaw \\ A major question in evolutionary biology is how mating patterns affect the fitness of offspring. However, in animals and seed \\ plants it is virtually impossible to investigate the effects of specific gamete genotypes. In bryophytes, haploid gametophytes \\ grow via clonal propagation and produce millions of genetically identical gametes throughout a population. The main goal of \\ this research was to test whether gamete identity has an effect on the fitness of their diploid offspring in a population of the \\ aquatic peat moss Sphagnum macrophyllum. We observed a heavily male-biased sex ratio in gametophyte plants (ramets) and \\ in multilocus microsatellite genotypes (genets). There was a steeper relationship between mating success (number of different \\ haploid mates) and fecundity (number of diploid offspring) for male genets compared with female genets. At the sporophyte \\ level, we observed a weak effect of inbreeding on offspring fitness, but no effect of brood size (number of sporophytes per \\ maternal ramet). Instead, the identities of the haploid male and haploid female parents were significant contributors to variance \\ in fitness of sporophyte offspring in the population. Our results suggest that intrasexual gametophyte/gamete competition may \\ play a role in determining mating success in this population.
}

Heredity (2016) 116, 523-530; doi:10.1038/hdy.2016.13; published online 24 February 2016

\section{INTRODUCTION}

A major focus in population biology is the estimation of mating success among individuals in a population and the consequences of parental mating patterns on offspring fitness (Fisher, 1930; Gustafsson, 1986; Merilä and Sheldon, 1999). In animals and most plants, 'parents' are defined as the diploid individuals that mate to produce diploid offspring. This conceptual formulation of parentage is limited because (except in severely inbred diploid individuals) the gametes they produce are genetically variable and will therefore have variable affects on offspring fitness. Although the variation can be studied in aggregate (as the result of recombination in the diploid parent), the expected fitness impact of individual gametes cannot be studied.

In agriculture, the genetic contribution of (diploid) parents to the fitness of their offspring is often deduced using controlled breeding experiments (Griffing, 1956). The 'general combining ability' of a parent is assessed through crosses with many mates (Falconer and Mackay, 1996). The genetic contribution of (diploid) parents to offspring is also studied in aggregate in sexual selection studies, where 'cryptic female choice' refers to apparent nonrandom mating success among haploid sperm in the female reproductive tract (Gasparini et al., 2010; see, for example, Firman and Simmons, 2010; Boschetto et al., 2011). In seed plants, pollen competition experiments frequently indicate that seed set (average) fitness increases with the number of pollen donors (Mulcahy and Mulcahy, 1975; Winsor et al., 2000; Zhang et al., 2010). Both examples suggest that intrasex competition at the haploid stage is important for determining offspring fitness (Snow and Spira, 1991; Marshall and Diggle, 2001), but the success of individual sperm or egg genotypes cannot be directly assessed.

From the perspective of a gamete, fecundity can be defined as the number of fertilization events the gamete participates in. For animals and seed plants, millions of genetically distinct gametes are produced by the diploid parent, and each gamete either participates in fertilization or not. As gametes in these organisms do not generally clonally replicate, 'parental' reproductive success is applied to diploid individuals rather than their haploid gametes (which are, more specifically, the actual parents). Gamete fecundity may be viewed as a binary trait in these organisms (that is, successful or not).

Because of their homosporous, haploid-dominant life cycle, bryophytes present a unique opportunity to study the effects of the haploid stage on mating success and offspring fitness. Individual haploid gametophytes produce thousands of genetically identical gametes via mitosis, and reproductive success of gamete genotypes is therefore quantitative rather than binary. In addition, clonal growth of the haploid stage before gamete production can result in genetically identical zygotes produced simultaneously throughout the population. These life-cycle features permit estimation of reproductive success for individual gamete genotypes, as well as the fitness consequences of specific haploid mating combinations.

In this study, we present an investigation of haploid parental contributions to fertilization success in a natural population of the peat moss Sphagnum macrophyllum, and the consequences of various gamete matings for zygote fitness. We sought to test the hypothesis 
that the identity of parental gamete genotypes affects the fitness of diploid offspring. Toward that end, we (1) assess variance in haploid fecundity (reproductive success) among individuals, (2) correlate haploid fecundity with the number of different mates for each individual and (3) determine the relative contribution of haploid parental genotype to diploid offspring fitness, compared with other factors including inbreeding depression and maternal resource limitation.

Our study population and the bryophyte life cycle allows us to study the 'Bateman gradient' in haploids for the first time. We calculated the mating success (number of genetically different mates) and fecundity (number of sporophyte offspring) for each maternal and inferred paternal genotype. In its original formulation, sexual selection was inferred from extreme secondary sexual characteristics, and defined by competition between individuals to mate and produce offspring (Darwin, 1859). The quantitative description of sexual selection later became synonymous with the relationship between fecundity (the number of offspring) and mating success (the number of different mates) (Bateman, 1948). When the strength of this relationship differs between the sexes, the sex with the reduced variance in mating success benefits from the ability to choose among many potential mates (Arnold, 1994). Our results demonstrate the utility of bryophytes to answer questions about reproductive biology that are intractable or impossible in seed plants or animals.

\section{MATERIALS AND METHODS}

\section{The bryophyte life cycle}

As with all land plants, bryophytes have an alternation of generations between a diploid spore-producing stage (sporophyte) and a haploid gamete-producing stage (gametophyte) (Figure 1). Both stages are multicellular and gametes are produced via mitosis from haploid gametophytes. Peat mosses (Sphagnum) are quasi-broadcast spawners; sperm are released into the water, whereas eggs are retained on female plants. The diploid sporophyte remains attached to the maternal gametophyte and is essentially a sphere containing, at maturity, meiotically produced spores. Although immature sporophytes are green, they lose their chlorophyll as they mature and are dependent on the maternal gametophyte for nourishment (Duckett et al., 2009). Before spore release the sporophyte is raised by a pseudopodium of maternal gametophyte tissue. Meiotically produced spores germinate and grow into large, persistent haploid gametophytes that can multiply via clonal propagation. Each independent gametophyte (ramet) may share an identical genotype with hundreds of other disconnected ramets-collectively, the haploid genet. Depending on the clonal structure of the population, genets may be represented by few to many independent ramets, and these ramets may be dispersed throughout the population.

In an idealized bryophyte population, let there be one male and one female genet. The (haploid) male genet undergoes vegetative propagation and each of the many ramets produce sperm by mitosis, giving rise to genetically identical sperm. The same is true of the (haploid) female clone that produces many genetically identical eggs. Fertilization between gametes produced by the one male clone and one female clone would produce a population of diploid sporophytes that are genetically identical because they result from fusion of the same male and female gamete genotypes. An important implication of this life cycle is that genetically equivalent fertilization events may be replicated in the population. In a genetically variable (multiclonal) population, the fecundity (fertilization success) of each male (and female) gametophyte genet need not be binary. Instead, haploid bryophyte fecundity is a quantitative trait. For example, one haploid male gametophyte may participate in one to many fertilization events, or none. This is in contrast to seed plants or animals, in which a gamete (or gametophyte) can participate in a maximum of one fertilization eventhaploid fecundity is binary.

The bryophyte life cycle also allows for unequivocal resolution of haploid parentage for diploid offspring. Because the sporophyte remains physically attached to the maternal gametophyte throughout its life (Figure 1), maternity is obvious and paternity can be inferred by subtracting the maternal haploid genotype from the diploid sporophyte genotype (Szövényi et al., 2009).

\section{Study site and species}

The study site is in the Hell Hole Bay Wilderness Area in the Francis Marion National Forest, Berkeley County, South Carolina (33.218 $\left.{ }^{\circ} \mathrm{N} 79.712^{\circ} \mathrm{W}\right)$. The sampling site is a break in the otherwise dense swamp pocosin: an open canopy of Taxodium trees standing in up to a meter of water. The understory contains several trees and shrubs including Nyssa biflora, Lyonia lucida and Vaccinium formosum. The aquatic herbaceous plants, Dulichium arundinaceum, Carex striata and Nymphaea odorata, occur scattered in the open water with several other peat moss species, including Sphagnum cuspidatum, S. magellanicum and $S$. portoricense. However, the dominant plant, floating in the water in nearly continuous mats covering an area of roughly one acre $\left(4000 \mathrm{~m}^{2}\right)$, is the aquatic peat moss S. macrophyllum.

S. macrophyllum is in the subgenus Subsecunda (Shaw et al., 2008a), and occurs in North America along the coastal plains of the Atlantic Ocean and Gulf of Mexico from Newfoundland to Texas (McQueen and Andrus, 2009). It usually grows in acidic waters such as roadside ditches, the margins of small ponds and in wet areas of pine savannahs, especially where there is underlying sandy soil (Anderson et al., 2009). Based on extensive field work throughout eastern North America, Hell Hole Swamp has the largest biomass of S. macrophyllum we have observed at a single site. It is also the only site where S. macrophyllum undergoes consistent and abundant sexual reproduction; the
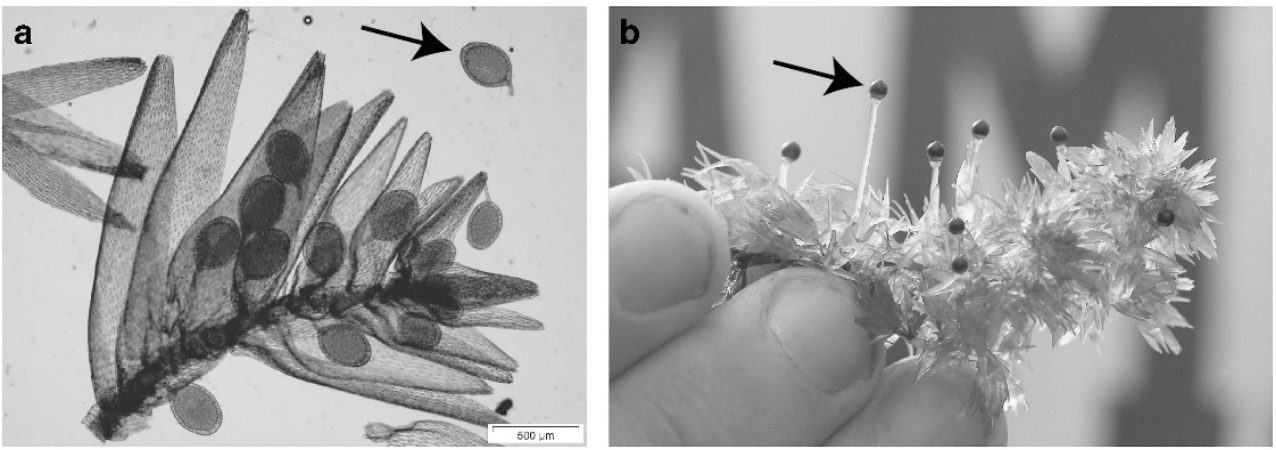

Figure 1 Reproductive structures in the peat moss S. macrophyllum, a species that has separate male and female haploid gametophytes. (a) In S. macrophyllum, male plants produce sperm in antheridia (arrow), of which at least 12 can be seen. The gametes are produced by mitosis from the perennial male plants; therefore, all sperm produced in all antheridia shown are genetically identical to each other, and to the male gametophyte that produced them. Sperm are released and swim through water to female plants to effect fertilization. Photo by MG Johnson. (b) A haploid female gametophyte (large leafy plant) can be identified by the presence of diploid sporophytes (arrow; small black spheres). This female ramet has a sporophyte brood of at least eight. Each sporophyte in this brood thus shares exactly half of its diploid genotype; genetic differences within the brood could only result from having different fathers. The translucent tissue that exerts each sporophyte is haploid, produced by the maternal gametophyte. Photo by B Shaw (Duke University, Durham, NC, USA). 
plants produced sporophytes every year since we began sampling the site in 2005 (MG Johnson et al., unpublished). Sporophytes are otherwise uncommon in this species.

\section{Reproductive phenology survey}

To describe the sex ratio and reproductive phenology of the population, we sampled gametophytes throughout a breeding season from December 2009 to June 2010. We established eight 'neighborhoods' throughout the population of floating mats of S. macrophyllum, with each neighborhood marked by a PVC pipe so that sampling would occur in the same area each month (for a schematic of the population, see Supplementary Figure 1). At each visit we sampled 12 gametophytes from within $1 \mathrm{~m}$ of the pipe, for a total of 96 plants sampled each month. We then assessed the sexual expression of gametophytes under a dissecting microscope.

S. macrophyllum, like all members of the subgenus Subsecunda, has unisexual gametophytes that produce either sperm (male) or eggs (female) but not both. There is little morphological sex differentiation, but male gametophytes are identified by antheridia (the male gametangia that produce sperm). Our observations indicate that antheridia are produced between December and March; plants are not identifiable as male outside this temporal range.

Female gametophytes are more challenging to identify in the field and require microscopic examination; archegonia (the female gametangia that produce eggs) are less conspicuous than are antheridia and often difficult to locate. Archegonia usually turn a reddish color after fertilization and this can help. The most reliable way to identify a gametophyte as female is by the presence of sporophytes because they remain attached to their maternal gametophyte. Females are identifiable beginning in late December, and young, macroscopic sporophytes are obvious by February. Sporophytes mature from April through June, and spores are released by July.

We characterized genetic diversity among male gametophytes as well as those gametophytes with no evidence of sexual expression ('nonexpressing'). However, male and female plants become sexually mature at different times, and because gametangia (or attached sporophytes in the case of females) are required to confirm gametophyte sex, we sampled plants throughout the breeding season from December through June. We marked 8 locations (neighborhoods) and randomly sampled 12 gametophytes (ramets) within $1 \mathrm{~m}^{2}$ at each neighborhood each month.

We determined the sex of each gametophyte by microscopic dissection, classifying each as 'male' (presence of antheridia), 'female' (presence of archegonia or attached sporophytes) or 'nonexpressing' (neither gametangia nor sporophytes observed). In order to determine whether female plants had already mated at the time of collection, all female gametophytes were transferred to individual tubes containing sterile water and observed for subsequent maturation of sporophytes. All gametophytes identified as females in the 2009-2010 breeding season $(N=126$, Table 1$)$ eventually produced sporophytes in the lab, indicating that each had already mated at the time of collection.

Table 1 Phenology of sexual expression and sporophyte production in S. macrophyllum at Hell Hole Swamp

\begin{tabular}{lrrcr}
\hline Month & Male & Female & Nonexpressing & Sporophytes \\
\hline December 2009 & 46 & 7 & 43 & 0 \\
January 2010 & 40 & 16 & 40 & 66 \\
February 2010 & 44 & 16 & 36 & 76 \\
March 2010 & 40 & 31 & 25 & 182 \\
April 2010 & 28 & 18 & 50 & 82 \\
May 2010 & 0 & 24 & 72 & 151 \\
June 2010 & 0 & 14 & 82 & 45 \\
Total & 198 & 126 & 348 & 602
\end{tabular}

A total of 96 plants were sampled at the same 8 locations (12 per location) within the population throughout one breeding season. Male plants were identified by the presence of antheridia (spermproducing structures) during microscopic dissection. Female plants were identified through the presence of archegonia (egg-producing structures) or sporophytes. Plants with no sexual structures of either type were recorded as nonexpressing.

\section{Sporophyte sampling and fitness proxy}

We sampled mature sporophytes over 2 years: April-May 2009 and March-June 2010. In 2009, sporophytes were sampled throughout the population; in 2010, we sampled mature sporophytes that were attached to the female gametophytes we sampled during the monthly phenology survey. Sphagnum sporophytes are roughly spherical capsules containing, at maturity, haploid spores (products of meiosis). The diameter of the capsule is a reliable predictor of spore number across multiple species in Sphagnum (Sundberg and Rydin, 1998). We measured the diameters of all sporophytes in the study when they were at full maturity and we use this measure as a proxy for sporophyte fitness (Szövényi et al., 2009). This phenotypic measurement allows for a simple estimate of potential reproductive output for the sporophyte without damaging the tissue necessary for genotyping the sporophyte.

\section{Genotyping}

Using material collected from the phenology survey, we genotyped every gametophyte identified as female (75 samples), a representative sample of male (58 samples), and nonexpressing (65 samples) gametophytes from each neighborhood each month. Sporophytes were sampled throughout the population in April and May 2009, in addition to all mature sporophytes sampled during the phenology survey in March through June 2010 (321 total sporophytes).

For DNA extractions of gametophyte tissue, a portion of the gametophyte's capitulum (the dense cluster of branches at the apex of Sphagnum plants) was removed. For sporophytes, the entire capsule was used. DNA extraction followed the cetyltrimethylammonium bromide protocol (Shaw et al., 2003). Briefly, tissue was flash frozen in tubes using liquid nitrogen and ground into a powder using steel beads. Cellulose and proteins were removed using cetyltrimethylammonium bromide and chloroform mixed with $\beta$-mercaptoethanol in a 24:1 ratio. DNA pellets were precipitated using cold isopropanol and eluted in $50 \mu \mathrm{l}$ of TE buffer. In preparation for PCR amplification, we diluted the DNA of gametophytes 7:1, and diluted sporophytes 2:1.

All plants were genotyped at 15 microsatellite loci, labeled as in Shaw et al. (2008b), 1, 4, 7, 9, 10, 14, 17, 18, 19, 20, 29, 65, 68, 75 and 93 , using previously described protocols.

\section{Paternity and genet assignment}

Because 'parentage' is defined at the haploid stage and because sporophytes remain attached to their maternal gametophyte, inferring the multilocus paternal haploid genotype is straightforward. Paternal alleles can be identified by simply subtracting the maternal haploid genotype from an attached sporophyte's diploid genotype. In the case of homozygous loci, the paternal and maternal genotypes were assumed to be identical. The inferred haploid paternal multilocus genotype for every sporophyte was determined using the custom Python script. The script determined the inferred paternal genotype by comparing the observed sporophyte genotype with its maternal haploid genotype. All sporophyte alleles not present in the maternal genotype were assumed to have originated in the paternal genotype. All of the sporophytes analyzed had an allele matching its maternal gametophyte, indicating that mutation rates at the microsatellite loci are minimal at this scale.

To characterize clonal structure of the population, a second Python script was used to assign every sample to unique multilocus genotypes. All haploid multilocus genotypes were included: female, male and nonexpressing gametophytes, as well as inferred paternal genotypes. The script first sorted samples with no missing data into separate multilocus genotypes. Samples with missing data were then reconciled with the initial multilocus genotypes and could have three outcomes: (1) the sample is unambiguously assigned to just one genotype, (2) the sample could be assigned to more than one genotype and (3) the sample represents a new genotype not seen previously. Samples were included in further analysis only if they unambiguously matched to exactly one unique multilocus genotype.

\section{Genetic analyses}

Population genetic statistics, including PhiPT (a multilocus equivalent of $F_{\mathrm{ST}}$ ), analysis of molecular variance and principal coordinate analysis, were calculated using GenAlEx version 6.5 (Peakall and Smouse, 2012). Missing data were not interpolated in any analysis using GenAlEx. 
For estimating diversity and inbreeding statistics, we needed to take into account that sporophytes attached to the same maternal gametophyte are not random draws from the population (that is, they share the same haploid mother). To partially address this bias, we generated 1000 'subsets' of each population using a modified version of the bootstrap method of Szövényi et al. (2009). Each subset contained a random draw of one sporophyte per (haploid) maternal brood. For instance, in a population with three maternal gametophytes (A, B and C), each bearing four sporophytes (numbered 1-4), a subset population would contain one sporophyte randomly drawn from each mother, such as (A1, B2, C3). We then repeated this procedure by randomly drawing another sporophyte (with replacement) from each mother and recalculating each statistic on this new subset, such as (A3, B2, C4). This was done a total of 1000 times, resulting in a range of values for each population statistic. $F_{I S}$ was calculated from the observed and expected heterozygosity of sporophytes within this subset, and this was repeated 1000 times to estimate a range of inbreeding coefficients for the whole population.

We calculated the 'Bateman gradient' for the haploid genotypes with a linear regression of fecundity versus mating success in each sex. A slope significantly different from zero indicates that more mates leads to more offspring, and can indicate the overall mating pattern for the population: polygamous, polyandrous or polygynous. We also compared the slopes using an analysis of covariance. If the slope of the relationship between fecundity and mating success significantly differs between the sexes, it suggests the presence of sexual selection.

We explored three main explanations for variance in fitness in the population of sporophytes. First, we calculated the relationship between heterozygosity (percentage of heterozygous loci) and fitness at the level of the individual sporophyte and at the level of the brood. Each statistic was calculated using the pseudo-population approach described above to avoid bias because of nonindependence of sporophytes attached to the same maternal gametophyte. Second, we investigated whether the number of sporophytes in a brood affected the average size of sporophytes in the brood. Third, we conducted an analysis of variance to determine whether maternal or paternal genet identity was a significant predictor of sporophyte fitness, using Type III sums of squares.

All statistical analyses were conducted in R (version 3.1, R Core Development Team, 2012). R and Python scripts used to generate these analyses are available at github.com/mossmatters/moss_popgen_scripts, and data tables containing the multilocus genotypes of gametophytes and sporophytes are available in the Dryad data repository (http://dx.doi.org/10.5061/dryad.qj3hn).

\section{RESULTS}

Hell Hole Swamp: phenology and genetic diversity

The phenology survey in S. macrophyllum in the 2010 breeding season revealed a heavily male-biased sex ratio (Table 1) at the ramet level.

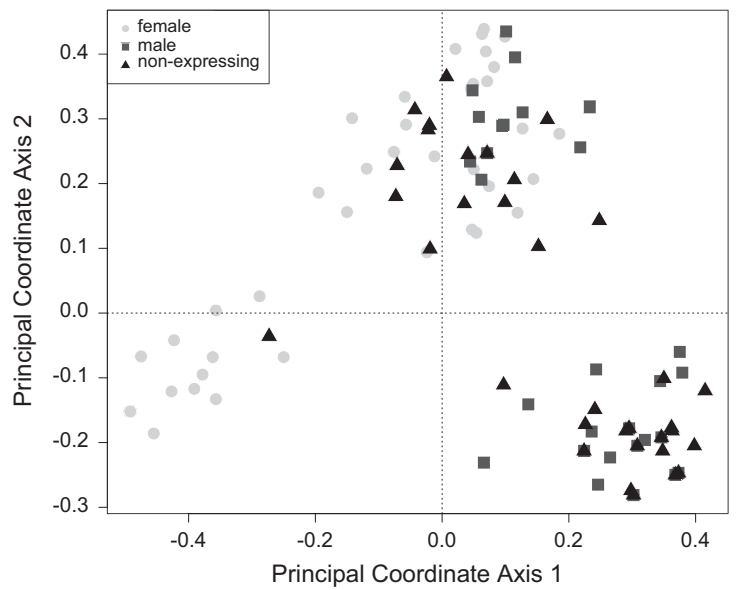

Male gametophytes could be identified (by the presence of antheridia) in 5 of the 7 months, and males always outnumbered females. In most months, a large percentage of gametophyte samples could not be determined as either male or female and were recorded as nonexpressing. Though this could reflect the greater difficulty of identifying female plants, the nonexpressing samples we genotyped most often matched male genotypes (see below). Overall, there were more male-expressing samples early in the breeding season, and female-expressing samples appeared later. However, the seven females identified in December 2009 all produced sporophytes when separately stored in sterile water, indicating that they were fertilized before collection. This observation, combined with field observations of exerted sporophytes in March, indicates that S. macrophyllum sporophytes require 3-4 months to fully mature.

Of the 197 gametophytes (ramets) genotyped, 149 were unambiguously assigned to one of 65 unique multilocus haploid genotypes (genets). In all, 17 genets included at least one demonstrably male ramet (identified by presence of antheridia) and 22 genets included at least 1 female ramet (identified by the presence of sporophytes). Of the nonexpressing ramets, 47 were unambiguously assigned to one genet-21 nonexpressing ramets matched a male genet whereas 8 ramets matched a female genet. The remainder of the nonexpressing ramets sorted into genets containing only nonexpressing individuals.

Among the female ramets, half (37 of 75) belong to a single genet that occurred in 6 of the 8 neighborhoods. The next most common genet contained just four ramets. One genet comprising 25 of 78 ramets dominated the male plants.

Male and female genets were genetically divergent (Figure 2). Female genets were significantly differentiated from male genets $(\mathrm{PhiPT}=0.103$, $P<0.001)$ and from nonexpressing genets (PhiPT $=0.039, P<0.05)$. Male genets were not differentiated from nonexpressing genets $(\mathrm{PhiPT}=0.00)$. A significant proportion of genetic differentiation among all ramets at the site was among neighborhoods $(\mathrm{PhiPT}=0.088$, d.f. $=7, P<0.01)$.

\section{Fecundity and mating success}

A total of 321 mature sporophytes were sampled and represent 10 unique maternal genets and 91 different inferred paternal genets. One single female, mentioned above, mated with 60 different paternal genets to produce $68.5 \%$ (220 of 321) of the sporophytes (Figure 3).

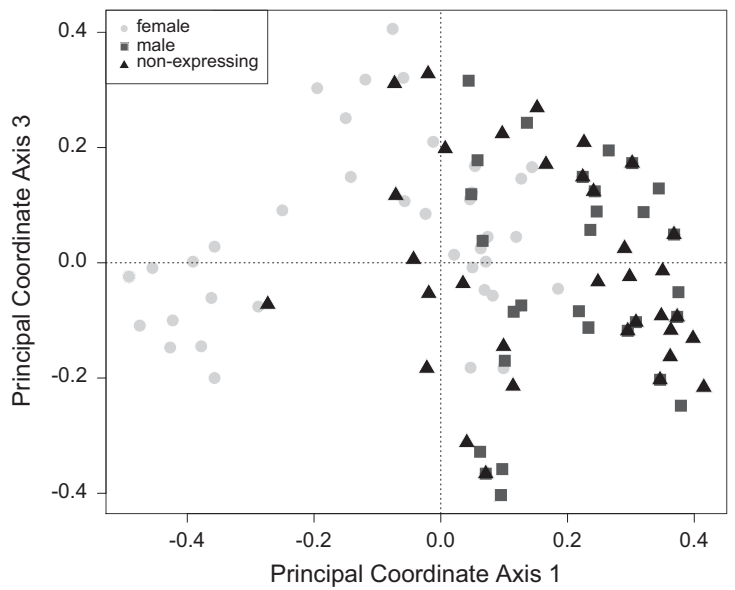

Figure 2 Principal coordinate analysis of genetic diversity in gametophytes. All gametophytes were classified as male (presence of antheridia) or female (presence of sporophytes). All other samples are classified as nonexpressing. When a nonexpressing ramet (triangles) has the same multilocus genotype as a male (squares) or female (circles) ramet, the points overlap. Percent of total variance: axis 1: $39.0 \%$; axis 2: $23.5 \%$; axis $3: 13.5 \%$. 
Each of the other maternal genets also mated with multiple paternal genotypes.

The skew in fecundity was also pronounced for the paternal genotypes (Figure 3). Three of the most commonly inferred genotypes together accounted for $36 \%$ of the sporophytes. Comparatively few of the inferred paternal genotypes matched male gametophytes we sampled: only 7 of the 91 inferred paternal genotypes match any gametophytes actually sampled in the population. A total of 19 inferred paternal genets were identified in at least 3 sporophytes; 6 of these fathers mated with more than one maternal genet.

The correlation between fecundity (number of offspring) and mating success (number of different genetic mates) was significant for both males $\left(r^{2}=0.644, P<2 \times 10^{-16}\right)$ and females $\left(r^{2}=0.983\right.$, $P<2.2 \times 10^{-8}$ ) (Figure 3). The slope of the male gradient was significantly steeper than the female gradient (male slope $=11.25$, female slope $=3.70$; analysis of covariance d.f. $=98, \quad F=63.14$, $\left.P<3.3 \times 10^{-8}\right)$. Both the relationship between mating success and fecundity for females, and the difference in slopes, remained significant after excluding the most common female (results not shown).

With the exception of the one very large female clone, there is no relationship between the number of ramets in a female genet and the number of mates. One female genet was identified from just one ramet but had sporophytes with eight different inferred fathers, whereas another genet identified from four ramets mated with only two different fathers. In addition, the relationship between the number of mates and the number of offspring is significant even when excluding the largest female clone. This suggests that size is not the only factor controlling mating success or fecundity.

\section{Sporophyte fitness}

The mean sporophyte size (our proxy for diploid offspring fitness) in the population was $1508 \mu \mathrm{m}( \pm 108 \mu \mathrm{m}$ s.d.). We investigated several sources of variance in sporophyte size: inbreeding, limitation of

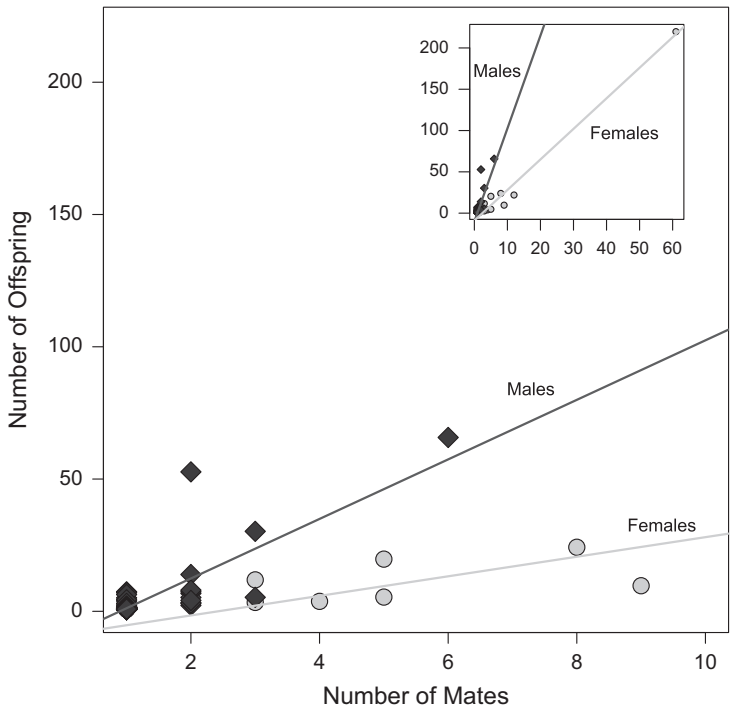

Figure 3 Bateman gradient for S. macrophyllum at Hell Hole Bay. The effect of mating success (number of different mates) on fecundity (number of sporophyte offspring) is shown for male (diamonds) and female (circles) gametophytes. The inset shows the full data set, including the very large female genet (top right), and the main plot is zoomed in to show the differentiation between male and female fecundity. Trend lines for each sex include all data points in both plots. resources from the maternal gametophyte and the genotype of the maternal and paternal gametophyte parents.

Using 1000 pseudoreplicate populations, the average $F_{I S}$ was -0.11 ( \pm 0.04 s.d.). In the three neighborhoods where sporophytes were most common, $F_{\text {IS }}$ was even more negative: neighborhood $3\left(F_{\mathrm{IS}}=-0.28, \pm 0.06\right.$ s.d. $)$, neighborhood $4\left(F_{\mathrm{IS}}=-0.34, \pm 0.05\right.$ s.d.) neighborhood 6 ( $F_{I S}=-0.14, \pm 0.02$ s.d.). The average multilocus heterozygosity was $54 \%$, and individual sporophytes ranged from 20 to $100 \%$ heterozygous loci.

There was a small but significant relationship between heterozygosity and sporophyte fitness (Figure 4a). The number of sporophytes attached to a female gametophyte ramet ('brood size') could limit the amount of maternal resources available to each sporophyte, potentially reducing the average fitness of the brood. However, there was no effect of brood size on the average brood fitness (Figure 4b, range 2-18, $\left.n=76, r^{2}=0\right)$.

Instead, the genetic identity of the maternal (d.f. $=5, F=4.24$, $P<0.01$ ) and paternal (d.f. $=22, \mathrm{~F}=3.58, P<0.001$ ) (haploid) parents explained much of the variance in sporophyte size (analysis of variance, Type III SSS, singletons removed). We detected no maternal $\times$ paternal interaction effect (d.f. $=8, \mathrm{~F}=1.13, P>0.1$ ), and paternal identity (9.2\%) accounted for greater variance than maternal identity $(3.6 \%)$. Because of low sample size, the effects of specific maternal or paternal genotypes could not be assessed.

To further investigate the effect of paternal identity on sporophyte fitness, we considered only the sporophytes attached to the most common female genet and classified the sporophytes based on paternal identity. In this analysis, all sporophytes in a category have the same haploid mother and the same haploid father, and therefore represent repeated fertilization events across the population. Despite considerable variation within each paternal group, there was a highly significant effect of haploid paternal identity on sporophyte fitness (Figure 5, analysis of variance, Type III SSS $\mathrm{F}_{16}=5.25, P<10^{-7}$ ).

\section{DISCUSSION}

\section{Phenology and genetic structure}

In contrast to the female-biased sex ratio found in most natural populations of bryophytes (reviewed in Bisang and Hedenäs, 2005), sexual expression in S. macrophyllum at Hell Hole Swamp is male biased (3:1 male/female ratio among sampled gametophytes). In other bryophytes, the low level of male sex expression has been attributed to a 'shy male hypothesis'; genetically male gametophytes do not always produce antheridia, obscuring an even sex ratio (Stark et al., 2010). If the sex ratio in our study population were truly even, then there must instead be the opposite effect- the presence of 'shy females' that are not producing archegonia or are not successfully fertilized. However, male and female gametophytes form distinct clusters on the principal coordinate analysis (Figure 2), and plants that we classified as nonexpressing are genetically distinct from the female, but not the male cluster. The high diversity of inferred paternal genotypes compared with the observed maternal genotypes also suggests sex bias at the genet level, although there may be a large unsampled pool of nonexpressing female genotypes.

Given the high amount of genetic diversity in the population, and the lack of a sex-specific genetic marker in Sphagnum, an even more comprehensive sampling of gametophytes would be required to determine whether the population is male biased at the genet level as well. However, it is worth mentioning that our sampled nonexpressing gametophytes were more likely to match the microsatellite profiles of male plants (21 nonexpressing ramets) than match genets including known females (8 nonexpressing ramets). Sex ratio bias 
a

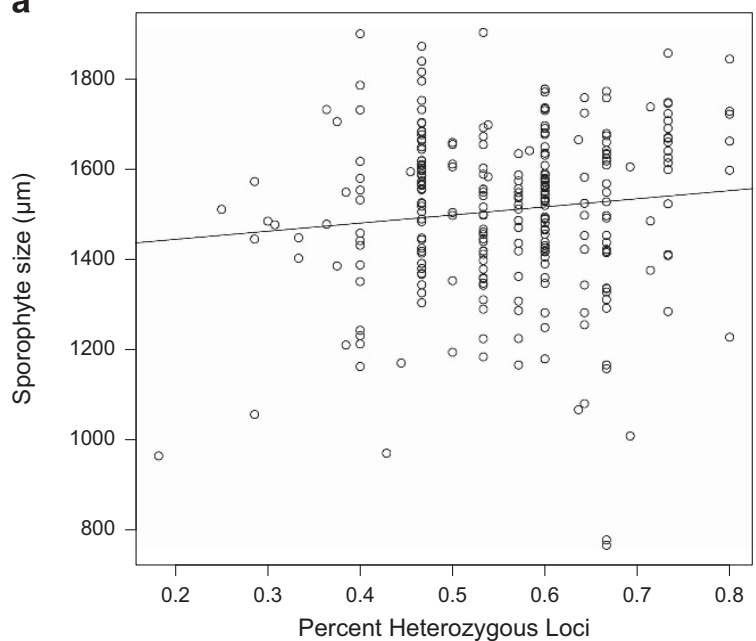

b

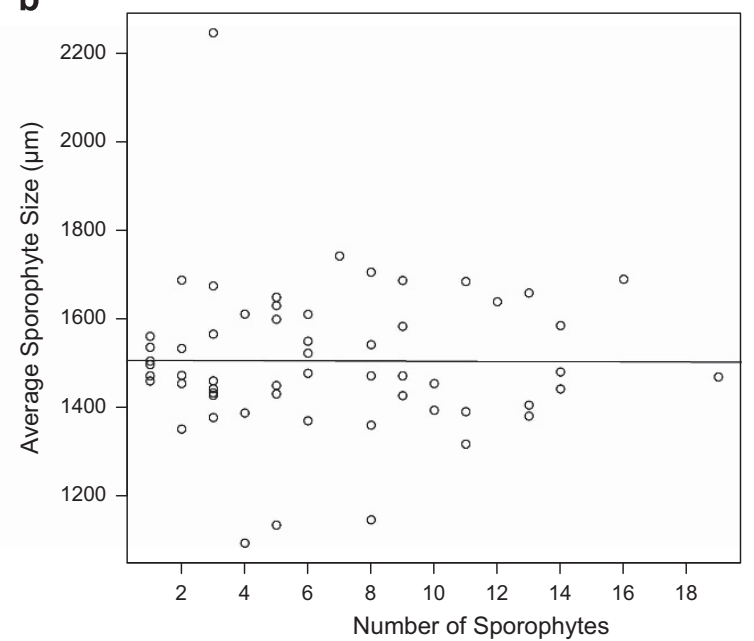

Figure 4 Effects of inbreeding depression and resource limitation on sporophyte fitness. (a) Relationship between percent heterozygosity and sporophyte size $\left(r^{2}=0.018, P<0.05\right)$. (b) Effect of 'brood size' (number of sporophytes supported by a maternal gametophyte) on average brood fitness $\left(r^{2}=0.00\right)$.

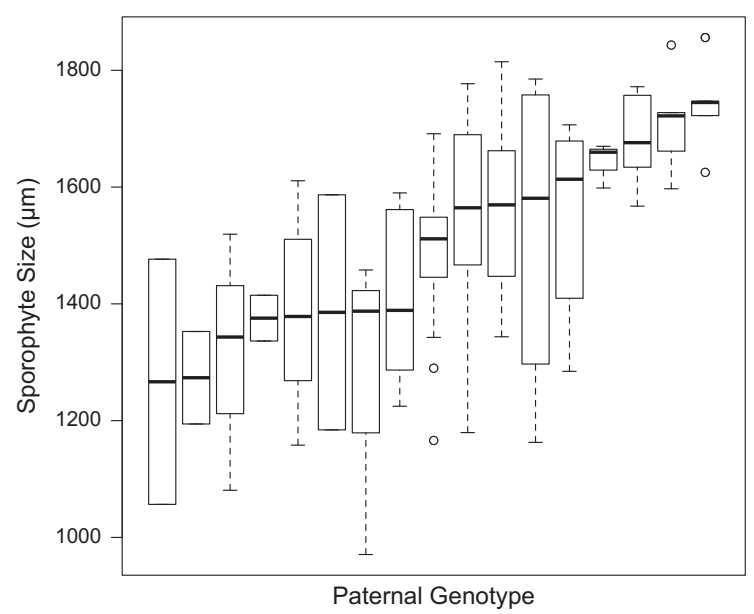

Figure 5 Distribution of sporophyte fitness by genetic father. All sporophytes shown have the same (haploid) genetic mother. Each box represents identical diploid sporophytes formed throughout the population; all variance within a box is environmental. All sporophytes within one column have the same haploid mother and the same haploid father, generating repeated fertilization events across the population. Despite the variance found within each box, there is a highly significant effect of paternal identity on sporophyte fitness for this subset (analysis of variance (ANOVA), $F=5.25$, d.f. $=16, P<10^{-7}$ ), indicating the effect of haploid paternal identity on sporophyte fitness must have a large genetic component.

among ramets in bryophytes may be a result of a bias in sex expression ('shy males'), a bias in growth rate between males and females or a result of biased spore death in sporophytes (Norrell et al., 2014). Distinguishing among these possibilities will require more detailed study at the level of sporelings and the development of sex-specific genetic markers for Sphagnum, as has been done with other mosses (Korpelainen et al., 2008; Hedenaes et al., 2010). Peat mosses are perennial plants that can reproduce asexually and hence the realized sex ratio bias in the population is likely to be stable over many years and breeding seasons. This is consistent with trends in seed plants, where sex ratio bias is more likely to be found in perennial plants (such as tree species) (Field et al., 2013).

A pronounced feature of sporophytes we sampled is an excess of heterozygosity, resulting in estimates of $F_{\text {IS }}$ that are significantly below zero. Using similar methods, Szövényi et al. (2009) also found a slightly negative $F_{I S}$ for Sphagnum lescurii. Other Sphagnum populations of species with unisexual gametophytes generally exhibit inbreeding coefficients below zero (Johnson and Shaw, 2015). Using isozymes, Eppely et al. (2007) found low (though positive) inbreeding coefficients in several other bryophytes with unisexual gametophytes. In sexually reproducing organisms with separate male and female sexes, small population sizes can lead to an excess of heterozygosity because of the effects of binomial sampling with small sample sizes (Rasmussen, 1979). Although considerable microsatellite variation exists among genets of $S$. macrophyllum, some may be a result of somatic mutations rather than sexual recombination. If this is true, then the true effective population size is very small. Because there is strong differentiation by sex in this population (Figure 2), fertilization events are likely to be from very different genotypes, resulting in highly heterozygous sporophytes. A broader survey of mating patterns in Sphagnum populations revealed that a highly negative $F_{I S}$ is especially common in populations of other aquatic species with unisexual gametophytes (Johnson and Shaw, 2015).

Sporophyte size does increase with heterozygosity in the study population (that is, evidence of inbreeding depression), but the effect is weak (Figure 4a). However, a large majority of sporophytes in this study had $>50 \%$ heterozygous loci. The excess of observed heterozygosity may result from homozygous sporophytes that did not reach maturity. In another aquatic species of subgenus Subsecunda there was a stronger relationship between heterozygosity and sporophyte fitness (Szövényi et al., 2009), but results from other unisexual species of Sphagnum suggest that inbreeding depression is not universal in Sphagnum (Johnson and Shaw, 2015).

\section{Variance in fecundity and mating success}

We found a high variance in the number of different mates, and the number of offspring they yield, for gametophytes of both sexes. Multiple paternity in sporophyte 'broods' raised by the same gametophyte mother was high, as has been previously described in S. lescurii (Szövényi et al., 2009). Unlike the previous study (where male gametophytes were monogamous or mated with few females), most paternal genotypes in S. macrophyllum mated with multiple female genets. Our study demonstrates abundant promiscuity in both sexes. 
The high variance in fecundity and mating success in both sexes provided an opportunity to test for the Bateman gradient among haploid plants in this population. The strength of the association in males compared with the association in females implies the underlying mating system for the population (Arnold and Duvall, 1994). In S. macrophyllum, there was a significant association between fecundity and mating success in both sexes (Figure 3 ). The greater slope of the gradient in male gametophytes suggests a polygamous mating system. In many other organisms, a significant difference in slopes between the sexes on the Bateman gradient has been interpreted as a signal of sexual selection (Levitan, 1998; Becher and Magurran, 2004; Tatarenkov et al., 2008; Anthes et al., 2010).

It is not clear what role, if any, sexual selection could play in a population of aquatic mosses. Sexual selection is expected to be most effective in mating systems where one sex has an upper fecundity limit that would put strong pressure on the other sex to compete for fertilization success (Arnold and Duvall, 1994). For S. macrophyllum, the Bateman gradient is linear for both sexes, although steeper for males (Figure 3). The trend in females is primarily because of one very large female genet that accounted for $50 \%$ of female ramets and $68 \%$ of sporophytes produced. Therefore, an alternative explanation for the relationship between fecundity and mating success is natural selection for clone size, rather than more direct competition for fertilization success within sexes.

There could also be sexual selection for clonal growth patterns. Given equivalent biomass, a clone that creates a large mate in one location may not contact as many potential mates as a clone that spreads outward and/or fragments. Clonal growth patterns are known to be extremely variable in $S$. cribrosum, the sister species to S. macrophyllum (Johnson et al., 2012). Given the higher slope of the Bateman gradient in males (Figure 3), there may be greater competition for exposure to multiple females as a mechanism to increase mating success and thus fecundity. In this study it was not possible to determine the effect of clone spatial breadth (vs clone size), because only a few of the male gametophytes sampled matched the inferred paternal genotypes.

Although purely speculative, the possibility that sexual selection could operate on gamete recognition in bryophytes is certainly feasible, given that sperm must swim to the eggs. Experimental tests could determine whether, as in seed plants (Snow and Spira, 1991) and sea urchins (Levitan, 2004), the number of available sperm donors affects fertilization success. In bryophytes, there may be competition between genetically identical sperm produced by the same genetic individual, and among male genotypes, an additional level of sperm competition not possible in other organisms. Experimental manipulation of sperm availability could test whether there is sperm competition, whether female genotypes choose among sperm, whether there is variance in chosen sperm genotypes and whether selection results in increased sporophyte fitness.

\section{Haploid parental effects on sporophyte fitness}

The strongest determinant of sporophyte fitness in the study population is the identity of the maternal and paternal haploid genotypes. This finding would be intractable or impossible to dissect in seed plants or animals, because it relies on the potential for quantitative fecundity in the haploid generation. The clonal propagation of gametophytes and the production of gametes via mitosis permits multiple, repeated fertilization events. In the most extreme case, one haploid female genet was fertilized by the same haploid male genet 55 times in 6 of the 8 neighborhoods. All of these diploid sporophytes are genetically identical; any size variance among these sporophytes must be environmental. Despite this, both maternal and paternal identity explained a significant proportion of sporophyte fitness variance.

This effect is in some ways analogous to general and specific combining ability in breeding experiments (Falconer and Mackay, 1996). A haploid male parent would have high general combining ability if, when crossed with multiple females, its offspring are on average more fit than the rest of the population. In quantitative genetics, this effect is important because it can be used to estimate the additive genetic variance in the population. Specific combining ability is the performance of one particular cross and represents nonadditive genetic variance. We did observe a higher relative fitness of certain males in the population when they crossed with the most common female genet (Figure 5). However, because this is a natural population and not a designed cross, we did not observe all possible combinations of males and females. This means there was not enough power to detect whether specific males or females produced larger offspring.

There is the potential for intergenome conflict among haploid parental genotypes in diploid sporophytes. Because all sporophyte offspring in Sphagnum are related to the maternal gametophyte equally, there could be selective pressure for maternal gametophytes to spread resources among all attached sporophytes. In contrast, there may be competition among the paternal genomes in a brood, with each attempting to maximize additional resources (Haig and Wilczek, 2006). The maternal gametophyte is responsible for supplying nutrients to young sporophytes (Duckett et al., 2009) and raises the capsule on a stalk of gametophyte tissue (Figure 1). Although nurturing sporophytes clearly presents resource demands on maternal gametophytes, there does not appear to be maternal resource limitation in this population. Though the large number of $S$. macrophyllum sporophytes (up to 18) found attached to some maternal ramets is extreme for Sphagnum (MG Johnson and AJ Shaw, unpublished), brood size does not seem to affect average brood fitness. It is possible that an effect may arise in other proxies for fitness not measured here, such as spore viability. Future experiments should follow the fitness consequences observed in the sporophyte to the next generation of gametophytes to determine the heritability of gametophyte fecundity.

\section{CONCLUSIONS}

We recorded, for the first time, how individual haploid genotypes (that is, gametes) vary in fertilization success and in fitness of their diploid offspring in a natural population. The identity of both maternal and paternal genotypes had significant affects on sporophyte size. We also found a relationship between the number of mates and the number of offspring, characteristic of a Bateman gradient suggesting the potential for sexual selection. Although it is unclear what traits may be selected, the extremely biased male sex ratio and the connection between gametophyte parental identity and sporophyte fitness both provide suggestive evidence that intrasexual competition for fertilization success occurs. To distinguish between natural and sexual selection, further experiments are necessary to determine the major life-history factors that determine fecundity, mating success and fitness. We have demonstrated the utility of the bryophyte system to provide unique windows into the often hidden haploid component of mating patterns in natural populations.

\section{DATA ARCHIVING}

$\mathrm{R}$ and Python scripts used to generate our analyses are available at github.com/mossmatters/moss_popgen_scripts, and data tables containing the multilocus genotypes of gametophytes and sporophytes are available in the Dryad data repository (http://dx.doi.org/10.5061/ dryad.qj3hn). Specimen vouchers from the reproductive phenology 
study, including the gametophytes selected for microsatellite genotyping, were deposited in the DUKE herbarium.

\section{CONFLICT OF INTEREST}

The authors declare no conflict of interest.

\section{ACKNOWLEDGEMENTS}

We thank R Johnson, M Ricca, L Clark, A Grusz and B Shaw for assistance in collecting the samples, S Boles and N Devos for help with microsatellite genotyping and C Fitzpatrick, I Liu and three anonymous reviewers for comments on earlier drafts of this manuscript. This research was supported by the Sigma-Xi Grant G200810150253 to MG Johnson and NSF Grant No. DEB-0918998 to AJ Shaw. The NSF grant was jointly awarded to AJ Shaw and B Shaw, but B Shaw was not involved in this study. B Shaw should still be listed as co-PI of this grant, which is documented here: http://www.nsf.gov/ awardsearch/showAward?AWD_ID=0918998.

Anderson L, Shaw B, Shaw AJ (2009). Peatmosses of the Southeastern United States. In Buck WR (ed). New York Botanical Garden: NY, USA.

Anthes N, David P, Auld JR, Hoffer JNA, Jarne P, Koene JM et al. (2010). Bateman gradients in hermaphrodites: an extended approach to quantify sexual selection. Am Nat 176: 249-263.

Arnold SJ (1994). Is there a unifying concept of sexual selection that applies to both plants and animals? Am Nat 144: S1-S12.

Arnold SJ, Duvall D (1994). Animal mating systems: a synthesis based on selection theory. Am Nat 143: 317-348.

Bateman AJ (1948). Intra-sexual selection in Drosophila. Heredity 2: 349-368.

Becher SA, Magurran AE (2004). Multiple mating and reproductive skew in Trinidadian guppies. Proc Biol Sci 271: 1009-1014.

Bisang I, Hedenäs L (2005). Sex ratio patterns in dioicous bryophytes re-visited. J Bryol 27 207-219.

Boschetto C, Gasparini C, Pilastro A (2011). Sperm number and velocity affect sperm competition success in the guppy (Poecilia reticulata). Behav Ecol Sociobiol 65: 813-821.

Darwin C (1859). The Origin of Species. John Murray: London.

Duckett JG, Pressel S, P'ng KMY, Renzaglia KS (2009). Exploding a myth: the capsule dehiscence mechanism and the function of pseudostomata in Sphagnum. New Phytol 183: 1053-1063.

Eppley SM, Taylor PJ, Jesson LK (2007). Self-fertilization in mosses: a comparison of heterozygote deficiency between species with combined versus separate sexes. Heredity 98: 38-44.

Falconer DS, Mackay T (1996). Introduction to Quantitative Genetics. 4th edn Pearson Prentice Hall: Harlow, UK.

Field DL, Pickup M, Barrett SCH (2013). Comparative analyses of sex-ratio variation in dioecious flowering plants. Evolution 67: 661-672.

Firman RC, Simmons LW (2010). Experimental evolution of sperm quality via postcopulatory sexual selection in house mice. Evolution 64: 1245-1256.

Fisher RA (1930). The Genetical Theory of Natural Selection. Clarendon Press: Oxford.

Gasparini C, Simmons LW, Beveridge M, Evans JP (2010). Sperm swimming velocity predicts competitive fertilization success in the green swordtail Xiphophorus helleri. PLoS One 5: e12146.

Griffing B (1956). Concept of general and specific combining ability in relation to diallel crossing systems. Aust J Biol Sci 9: 463-493.
Gustafsson L (1986). Lifetime reproductive success and heritability: empirical support for Fisher's fundamental theorem. Am Nat 128: 761-764.

Haig D, Wilczek A (2006). Sexual conflict and the alternation of haploid and diploid generations. Philos T Roy Soc B 361: 335-343.

Hedenaes L, Bisang I, Korpelainen H, Cronholm B (2010). The true sex ratio in European Pseudocalliergon trifarium (Bryophyta: Amblystegiaceae) revealed by a novel molecular approach. Biol J Linn Soc 100: 132-140.

Johnson MG, Shaw AJ (2015). Genetic diversity, sexual condition, and microhabitat preference determine mating patterns in Sphagnum (Sphagnaceae) peat-mosses. Biol J Linn Soc 115: 96-113.

Johnson MG, Shaw B, Zhou P, Shaw AJ (2012). Genetic analysis of the peatmoss Sphagnum cribrosum (Sphagnaceae) indicates independent origins of an extreme infraspecific morphology shift. Biol J Linn Soc 106: 137-153.

Korpelainen H, Bisang I, Hedenäs L, Kolehmainen J (2008). The first sex-specific molecular marker discovered in the moss Pseudocalliergon trifarium. J Hered 99: 581-587.

Levitan D (1998). Does Bateman's principle apply to broadcast-spawning organisms? Egg traits influence in situ fertilization rates among congeneric sea urchins. Evolution 52: 1043-1056.

Levitan D (2004). Density-dependent sexual selection in external fertilizers: variances in male and female fertilization success along the continuum from sperm limitation to sexual conflict in the sea urchin Strongylocentrotus franciscanus. Am Nat 164: 298-309.

Marshall DL, Diggle PK (2001). Mechanisms of differential pollen donor performance in wild radish, Raphanus sativus (Brassicaceae). Am J Bot 88: 242-257.

McQueen CB, Andrus RE (2009). Sphagnaceae. In: Flora of North America Editorial Committee (ed), Flora of North America North of Mexico. vol. 27. Oxford: New York, NY, USA.

Merilä J, Sheldon BC (1999). Genetic architecture of fitness and nonfitness traits: empirical patterns and development of ideas. Heredity 83: 103-109.

Mulcahy D, Mulcahy G (1975). The influence of gametophytic competition on sporophytic quality in Dianthus chinensis. Theor App/ Genet 46: 277-280.

Norrell TE, Jones KS, Payton AC, McDaniel SF (2014). Meiotic sex ratio variation in natural populations of Ceratodon purpureus (Ditrichaceae). Am J Bot 101: 1572-1576.

Peakall R, Smouse PE (2012). GenAIEx 6.5: genetic analysis in Excel. Population genetic software for teaching and research-an update. Bioinformatics 28: 2537-2539.

Rasmussen DI (1979). Sibling clusters and genotypic frequencies. Am Nat 113: 948-951.

Shaw AJ, Boles SB, Shaw B (2008a). A phylogenetic delimitation of the 'Sphagnum subsecundum complex' (Sphagnaceae, Bryophyta). Am J Bot 95: 731-744.

Shaw AJ, Cao T, Wang L, Flatberg KI, Flatberg B (2008b). Genetic variation in three Chinese peat mosses (Sphagnum) based on microsatellite markers, with primer information and analysis of ascertainment bias. Bryologist 111: 271-281.

Shaw AJ, Cox CJ, Boles SB (2003). Polarity of peatmoss (Sphagnum) evolution: who says bryophytes have no roots? Am J Bot 90: 1777-1787.

Snow A, Spira T (1991). Pollen vigour and the potential for sexual selection in plants. Nature 352: 796-797.

Stark LR, McLetchie DN, Eppley SM (2010). Sex ratios and the shy male hypothesis in the moss Bryum argenteum (Bryaceae). Bryologist 113: 788-797.

Sundberg S, Rydin H (1998). Spore number in Sphagnum and its dependence on spore and capsule size. J Byol 20: 1-16.

Szövényi P, Ricca M, Shaw AJ (2009). Multiple paternity and sporophytic inbreeding depression in a dioicous moss species. Heredity 103: 394-403.

Tatarenkov A, Healey CIM, Grether GF, Avise JC (2008). Pronounced reproductive skew in a natural population of green swordtails, Xiphophorus helleri. Mol Ecol 17: 4522-4534.

Winsor J, Peretz S, Stephenson A (2000). Pollen competition in a natural population of Cucurbita foetidissima (Cucurbitaceae). Am J Bot 87: 527-532.

Zhang C, Tateishi N, Tanabe K (2010). Pollen density on the stigma affects endogenous gibberellin metabolism, seed and fruit set, and fruit quality in Pyrus pyrifolia. J Exp Bot 61: 4291-4302.

Supplementary Information accompanies this paper on Heredity website (http://www.nature.com/hdy) 